\title{
Institutional experience using interstitial brachytherapy for the treatment of primary and recurrent pelvic malignancies
}

\author{
Paul Aridgides, MD**, Benjamin Onderdonk, MD*, Mary Cunningham, MD², Emily Daugherty, MD', Lingyun Du, MD!, \\ W. Douglas Bunn, MD², Rinki Agarwal, MD², Seung Shin Hahn, MD' \\ 'Department of Radiation Oncology, ${ }^{2}$ Department of Obstetrics and Gynecology, SUNY Upstate Medical University, Syracuse, NY, USA \\ *Authors contributed equally to this work.
}

\begin{abstract}
Purpose: The study assessed the outcomes of patients at a single institution with locally advanced primary and recurrent pelvic malignancies treated with interstitial high-dose-rate (HDR) or low-dose-rate (LDR) brachytherapy (BT), using a modified Syed-Neblett template.

Material and methods: Between 1996 and 2010, 60 patients with primary or recurrent pelvic malignancies were treated with interstitial BT. Thirty three patients had primary malignancies with $6.1 \%$ being stage I, $33.3 \%$ stage II, $45.5 \%$ stage III, and $15.2 \%$ stage IV; the remaining 27 patients were recurrent malignancies. Fifty four patients received external beam radiotherapy (EBRT) as part of their treatment course. The median EBRT, BT, and EBRT + BT doses were $45 \mathrm{~Gy}, 20 \mathrm{~Gy}$, and $65 \mathrm{~Gy}$, respectively. Thirty eight patients received concurrent chemotherapy with EBRT. Complete response (CR) was defined by absence of clinical and radiographic disease on first follow-up. Toxicity was graded as per Common Terminology Criteria for Adverse Events, version 4.0.

Results: The median follow-up was 37 months (4-234 months) and initial CR was achieved in 91\%. For primary cancers at diagnosis, 5-year local control (LC), 5-year progression-free survival (PFS), 5-year overall survival (OS) were $65 \%, 64 \%$, and $42 \%$ respectively. For recurrent cancers at diagnosis, 5-year LC, 5-year PFS, and 5-year OS were $80 \%, 51 \%$, and $37 \%$, respectively. There was a significant difference in both OS and PFS among different tumor sites $(p<0.05)$, with vaginal cancers having the best 5-year OS (55\%) and PFS (84\%). There was a total of 1 acute toxicity $\geq$ grade 3,6 late grade 3 toxicities, and late grade 4 toxicity.

Conclusions: Our series suggests that interstitial BT using a modified Syed-Neblett template is a safe and effective treatment for primary or recurrent pelvic malignancies. This technique allowed effective LC and $97 \%$ of patients had preservation of both bladder and rectal function.
\end{abstract}

Key words: endometrial, interstitial brachytherapy, recurrent tumors, vaginal.

\section{Purpose}

Brachytherapy (BT), often in conjunction with external beam radiation therapy (EBRT), is an integral component of definitive radiotherapy for treatment of gynecologic malignancies. Brachytherapy is essential for management of primary vaginal cancer where radical surgery is often precluded by significant morbidity [1,2]. Patients with vaginal recurrence of pelvic tumors such as endometrial cancer are infrequently candidates for repeat surgical excision [3], however combination BT and EBRT provides excellent long-term control and is a potentially curative option in localized disease $[4,5,6]$. Interstitial BT allows for treatment of deeper tumors that would be insufficient- ly covered by vaginal cylinder intracavitary BT, which is best suited for superficial $(\leq 0.5 \mathrm{~cm}$ thick) lesions and for adjuvant treatment following hysterectomy [7,8,9]. A perineal template is commonly employed to guide placement of afterloading needles that are directly inserted into the vagina and surrounding tissues, with particular attention to the location of normal structures including the rectum, urethra, and bladder [10]. Computed tomography (CT)based planning facilitates delivery of adequate dose to tumor volume within acceptable dose limits for adjacent critical organs $[11,12]$. Here we report our institutional experience using interstitial BT with a modified Syed-Neblett template (Elekta, Stockholm, Sweden) for the treatment of primary and recurrent pelvic malignancies. 


\section{Material and methods}

\section{Patient characteristics}

Our institutional series compromises 60 female patients receiving interstitial BT using a modified SyedNeblett template between 1996 and 2010. The patient population is detailed in Table 1. Patients had tumors of the female reproductive tract except for one patient with rectal cancer, which was included due to recurrence in the rectovaginal septum, and therefore amenable to interstitial BT with this technique. All patients had pathologic confirmation of primary or recurrent disease. Staging included

Table 1. Patient characteristics of the brachytherapy study population

Factors

Number of patients (\%)

\begin{tabular}{|c|c|}
\hline \multicolumn{2}{|l|}{ Age, years } \\
\hline Median: 61 & - \\
\hline Range: $35-86$ & - \\
\hline \multicolumn{2}{|l|}{ Brachytherapy indication } \\
\hline Initial diagnosis ${ }^{a}$ & $33(55 \%)$ \\
\hline Stage I & 2 \\
\hline Stage II & 11 \\
\hline Stage III & 15 \\
\hline Stage IV & 5 \\
\hline Recurrent, no previous radiation ${ }^{b}$ & $21(35 \%)$ \\
\hline Recurrent, previous radiation ${ }^{c}$ & $6(10 \%)$ \\
\hline \multicolumn{2}{|l|}{ Primary site } \\
\hline \multicolumn{2}{|l|}{ Initial diagnosis } \\
\hline Vagina & 20 \\
\hline Cervix & 11 \\
\hline Vulva & 2 \\
\hline \multicolumn{2}{|l|}{ Recurrent, no previous radiation } \\
\hline Uterus & 13 \\
\hline Cervix & 4 \\
\hline Ovary & 2 \\
\hline Vulva & 2 \\
\hline \multicolumn{2}{|l|}{ Recurrent, previous radiation } \\
\hline Uterus & 3 \\
\hline Cervix & 1 \\
\hline Vulva & 1 \\
\hline Rectum & 1 \\
\hline
\end{tabular}

aInitial diagnosis - BT as a component of primary treatment; ${ }^{b}$ Recurrent, no previous radiation - BT for recurrent disease in patients without prior pelvic radiation; 'Recurrent, previous radiation - BT for recurrent disease in patients who had previously received pelvic RT physical examination by radiation oncology and gynecology oncology, pelvis CT and/or pelvic MRI (magnetic resonance imaging), and either whole body PET (positron emission tomography), chest $\mathrm{CT}$, or chest $\mathrm{X}$-ray to assess for distant disease. The majority of patients received interstitial BT as a component of initial therapy (55\%) or for recurrent disease after surgery $(35 \%)$. The vagina was the most common primary site for patients treated with BT at diagnosis (61\%). Uterine cancer compromised the majority of patients $(63 \%)$ treated for recurrent disease without previous radiation. Only six patients $(10 \%)$ received BT for recurrence after prior radiation. Follow-up schedule consisted of physical examinations 1 month following BT, every 3 months years $1-2$, every 6 months for $3^{\text {th }}-5^{\text {th }}$ year, and then yearly. They were supplemented with surveillance imaging (CT, PET/CT) until resolution or stability of abnormal findings.

\section{Brachytherapy procedure}

Interstitial BT was performed under general anesthesia with the use of a modified Syed-Neblett template. Prior to the procedure, the anticipated needle locations were determined using physical exam and CT/MRI imaging. The template was secured using a vaginal obturator and with sutures placed in the skin of the perineum/vulva. Needles were directed for adequate volumetric coverage of residual tumor on physical exam and all initially involved areas detected by physical exam and 3-dimensional (CT or MRI) imaging. Digital rectal exam was performed to ensure no posterior needles were placed in the rectum. Needles were placed by one radiation oncologist (SH). Needles were marked pre-procedure to the appropriate depth of insertion determined on pre-implantation review of $\mathrm{CT} / \mathrm{MRI}$ imaging. Flouroscopic verification confirmed needle placement and geometry without the routine use of ultrasound guidance. For select cases with significant concern for bowel injury, needles were placed under laparoscopic guidance. There were no cases of bowel perforation or significant bleeding. A median of 15 (range: 6-35) afterloading catheters were used. Each BT course consisted of a single Syed-Neblett implantation followed by inpatient hospital stay for the duration of BT.

A CT scan was obtained for 3-dimensional dosimetric planning (BrachyVision, Varian, Palo Alto, CA, USA) after contouring of targets and normal tissue structures (bladder, rectum, urethra). The gross tumor volume (GTV) was defined as residual tumor at the time of BT detectable by physical exam or imaging (CT or MRI). The clinical tumor volume (CTV) was defined as all initially involved primary tumor areas detected by physical exam or imaging (CT or MRI) at presentation. Dose was prescribed to ensure tumor coverage to both the GTV and CTV.

High-dose-rate (HDR) brachytherapy, employing ${ }^{192}$ Ir sources and a remote afterloader (VariSource, Varian, Palo Alto, CA, USA), was utilized in the majority $(82 \%)$ of patients. The median HDR fractionation was 5 Gy (range: 2.5-5 Gy), which was given twice daily with minimum 6 hour interval between fractionations. The median total BT dose was 20 Gy (range: 10-40 Gy). An additional 11 patients (18\%) were treated with low- 
dose-rate (LDR) brachytherapy using ${ }^{192}$ Ir. For LDR, the median prescribed BT dose was 30 Gy (range: 20-40 Gy), and the median dose/hour was $0.5 \mathrm{~Gy} / \mathrm{hr}$ (range: 0.4-0.8 Gy/hr). Typical treatment scheme was $45 \mathrm{~Gy}$ EBRT to the pelvis followed by 20-25 Gy BT and was dependent on individual patient and disease characteristics, including previous external beam radiation and dose to critical organs. The median total equieffective total dose in 2 Gy fractions (EQD2) for HDR patients was 74.3 Gy (range 64.3-84.3 Gy). Higher BT dose of 30 Gy following 45 Gy EBRT (EQD2 of $84.3 \mathrm{~Gy}$ ) was intended for patients with cervical cancer and patients with significant residual disease (suboptimal response to EBRT). Treatment characteristics are summarized in Table 2.

Low-dose-rate brachytherapy, utilizing ${ }^{192}$ Ir ribbons with sources spaced $1 \mathrm{~cm}$ apart, was used in the remainder $(18 \%)$ of patients. This corresponded to the earliest cohort of patients prior to departmental conversion to HDR. A pre-plan (Quimby method) was generated using $\mathrm{CT} / \mathrm{MRI}$ imaging to ensure sufficient quantity of ribbons were ordered to cover the CTV/GTV to prescribed dose over appropriate time (30 Gy over 2-3 days). After catheter insertion under general anesthesia, CT based planning was used to develop a plan where more than $90 \%$ of the

Table 2. Brachytherapy treatment characteristics, including dose rate (high-dose-rate, low-dose-rate), inclusion of external beam radiation, disease status (initial diagnosis, recurrent without previous radiation, recurrent after previous radiation), the use of systemic chemotherapy with external beam radiation, and external beam field

\section{Brachytherapy treatment characteristics}

\begin{tabular}{|c|c|c|}
\hline & & \\
\hline HDR & $49(82)$ & 20 Gy (10-40) \\
\hline Fractionation, BID & - & 5 Gy $(2.5-5)$ \\
\hline Initial diagnosis ${ }^{a}$ & 27 & 20 Gy (10-40) \\
\hline Recurrent, no previous $\mathrm{RT}^{\mathrm{b}}$ & 17 & 20 Gy (16-30) \\
\hline Recurrent, previous $\mathrm{RT}^{\mathrm{c}}$ & 5 & 20 Gy (16-33.6) \\
\hline LDR, $n=11(18 \%)$ & $11(18)$ & 30 Gy (20-40) \\
\hline Dose rate & - & 0.5 Gy/hour (0.4-0.8) \\
\hline Initial diagnosis & 6 & 32.5 Gy (20-40) \\
\hline Recurrent, no previous RT & 4 & 25 Gy (20-30) \\
\hline Recurrent, previous RT & 1 & 33.6 Gy \\
\hline EBRT with BT boost & $54(90)$ & 45 Gy (20-63) \\
\hline Initial diagnosis & $33(100)$ & 45 Gy (41.4-63) \\
\hline Concurrent EBRT/chemotherapy & $27(82)$ & - \\
\hline Recurrent, no previous RT & $20(95)$ & 45 Gy (20-59) \\
\hline Concurrent EBRT/chemotherapy & $10(50)$ & - \\
\hline Recurrent, previous RT & $1(17)^{d}$ & 30 Gy \\
\hline Concurrent EBRT/chemotherapy & $1(100)$ & - \\
\hline \multicolumn{3}{|l|}{ EBRT fielde } \\
\hline Pelvis, whole & $51(94)$ & 45 Gy (41.4-51) \\
\hline Pelvis alone & $26(51)$ & 45 Gy (41.4-50.4) \\
\hline Pelvis, inguinal & $13(25.5)$ & 51.7 Gy (41.4-60) \\
\hline Pelvis, para-aortic & $3(5.5)$ & 45 Gy (45-60) \\
\hline Pelvis then boost & 9 (18) & 50.4 Gy (39.6-63) \\
\hline Primary only & $3(6)$ & 30 Gy (20-50) \\
\hline
\end{tabular}

$H D R$ - high-dose-rate, $L D R$ - low-dose-rate, BID - twice daily radiation, $R T$ - radiation therapy, BT-brachytherapy, EQD2 - equieffective total dose in 2 Gy fractions $(\alpha / \beta=10)$ of brachytherapy plus external beam radiation therapy, EBRT - external beam radiation therapy

a Initial diagnosis - BT as component of primary treatment, ${ }^{b}$ Recurrent, no previous radiation - BT for recurrent disease in patients without prior pelvic radiation, ${ }^{C}$ Recurrent, previous radiation - BT for recurrent disease in patients who had previously received pelvic RT, ${ }^{d}$ One out of 6 patients with previous EBRT received reirradiation with EBRT to $30 \mathrm{~Gy}$ and $16 \mathrm{~Gy} B T$ boost (4 Gy $\times 4, B I D$ ), all others received BT alone, ${ }^{e} E B R T$ field and total dose (including boost) for patients receiving whole pelvis with or without coverage of inguinal lymph nodes or para-aortic lymph nodes, or for primary site only 
tumor targets are covered with prescription dose, with 70 Gy maximum to rectum and urethra, and 75 Gy maximum to bladder. The selected catheters are then loaded in the patient's inpatient room, which has proper shielding, and after the appropriate treatment duration, both the iridium ribbons and then the template are removed.

\section{Outcomes analysis}

Overall survival was calculated from the time of initial diagnosis to death or last follow-up. Progression free survival was calculated from the date of completion of therapy to diagnosis of recurrence. Recurrence was classified as local, locoregional, and distant progression of disease. Complete response was defined by absence of residual disease on examination and radiographic evaluation by CT or PET typically one to three months following completion of therapy. Acute and late toxicities of the vagina, bladder, and rectum were assessed and graded as per Common Terminology Criteria for Adverse Events v. 4.0 [13]. The 5-year local control, 5-year progression-free survival, and 5-year overall survival were assessed using the Kaplan-Meier method. The impact of various prognostic factors such as patient age at diagnosis, stage, tumor primary location, and primary versus recurrent tumors were assessed using a modified log rank test. Differences were statistically significant for $p$ values $<0.05$.

\section{A}

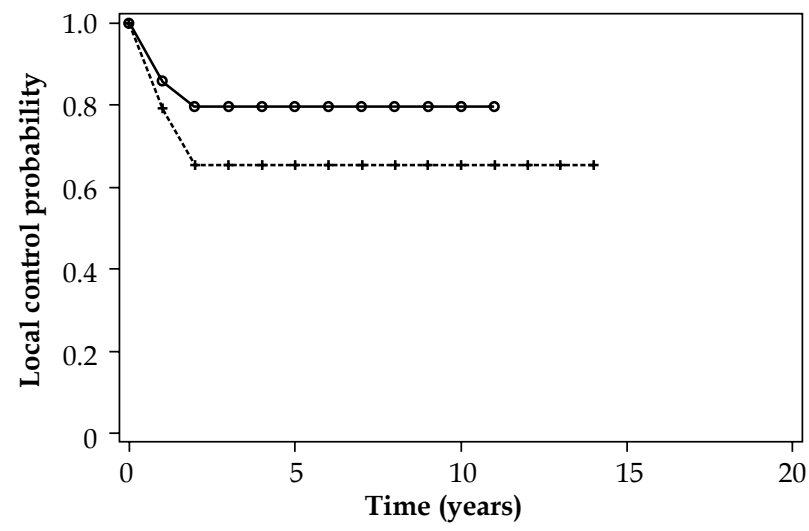

--+- Primary $\rightarrow$ Recurrent

C

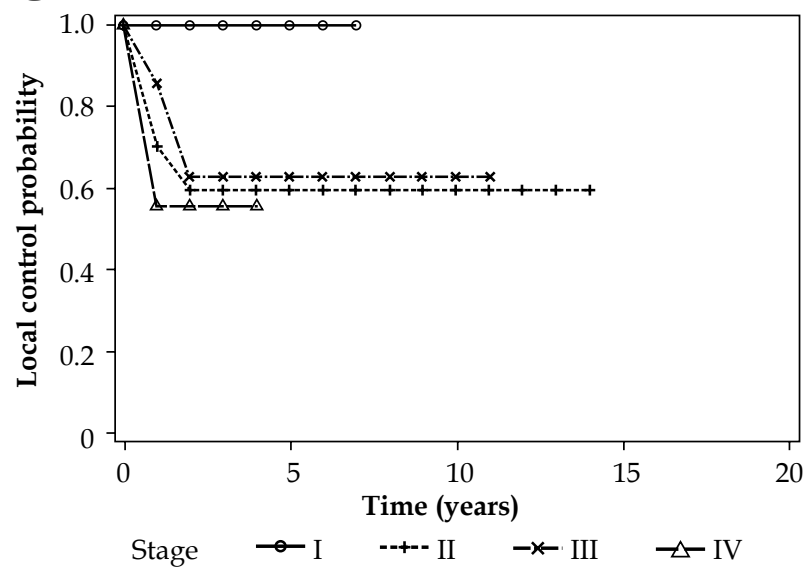

\section{Results}

\section{Local control}

Five patients were only evaluable for overall survival, and therefore excluded from additional analyses. The median follow-up was 36.9 months (range: 4-234 months) for the remaining 55 patients. Complete response was achieved in 50 patients $(91 \%)$ and 5 patients had persistent disease. Of the 50 patients achieving an initial complete response, 17 subsequently developed recurrence of which 6 included local failures. The median time to local failure was 9.6 months (range: 5.7-14.7 months).

For primary and recurrent malignancies treated with interstitial BT, there was no difference observed in local control rates (see Fig. 1A). The 5-year local control rate for primary tumors was $65 \%$ and $80 \%$ for recurrent tumors $(p>0.05)$. However, when primary tumor site was analyzed (see Fig. 1B), patients with uterine cancer had improved local control with no local failures seen $(p<0.05)$. For patients treated at initial diagnosis, earlier cancer stage exhibited a trend towards improved local control $(p=0.05$, see Fig. $1 C)$.

\section{Progression free and overall survival}

The five-year progression-free survival (PFS) for primary and recurrent malignancies was $64 \%$ and $51 \%$, re-

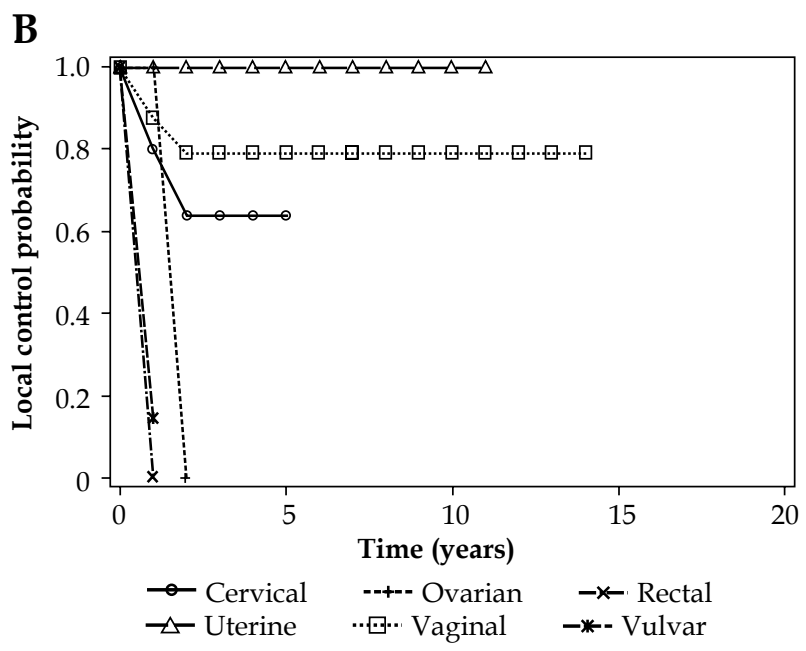

Fig. 1. Local control in patients receiving interstitial brachytherapy according to primary or recurrent tumors (A), primary site (B), and stage (I-IV) (C) 
spectively ( $p>0.05$, see Fig. 2A). Vaginal cancers exhibited a superior 5 -year PFS of $84 \%(p<0.05$, see Fig. 2B). There was no difference in PFS according to cancer stage ( $p>0.05$, see Fig. 2 C). The median overall survival was 34.6 months for all patients (range: 4 -234 months). The 5-year overall survival for primary and recurrent malignancies was $42 \%$ and $37 \%$, respectively ( $p>0.05$, see Fig. $3 \mathrm{~A})$. In the same way as vaginal cancers had a superior progression-free survival, they also exhibited a superior five-year overall survival of 55\% $(p<0.05$, see Fig. 3B). There was no difference observed in overall survival (OS) according to cancer stage $(p>0.05$, see Fig. 3C).

\section{Dosimetry}

Unfortunately, due to a software upgrade resulting in loss of data, digital review of dosimetry plans was not possible for the majority of patients. The maximum organ point dose was available in 37 patients $(76 \%)$ who received HDR BT and in 4 patients (44\%) treated with LDR BT. For HDR BT, the median bladder point dose was 3.41 Gy per fraction (range 1.71-5.9 Gy) and the median rectum point dose was 3 Gy per fraction (range 0.98$5.93 \mathrm{~Gy}$ ). For LDR BT, the median bladder point dose was 31 Gy (range 10.8-38.5 Gy) and the median rectum point dose was 18.9 Gy (range 11.0-30.5 Gy).

\section{A}

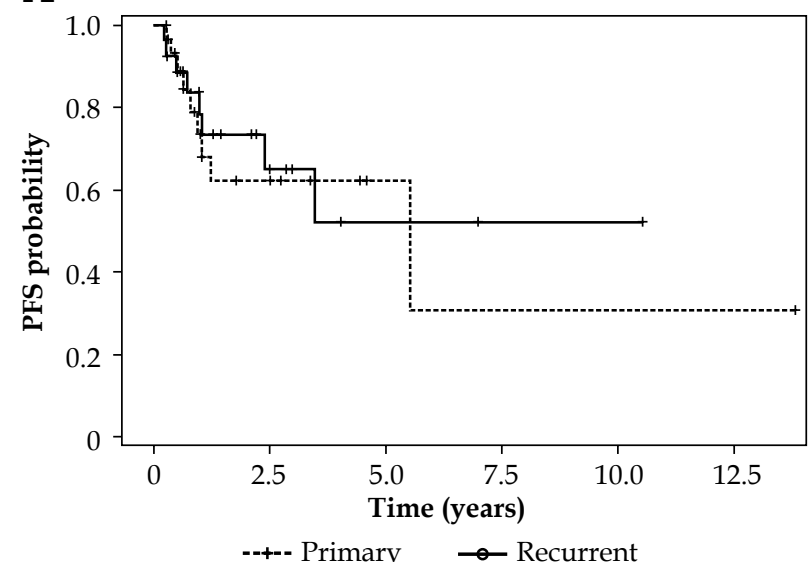

C

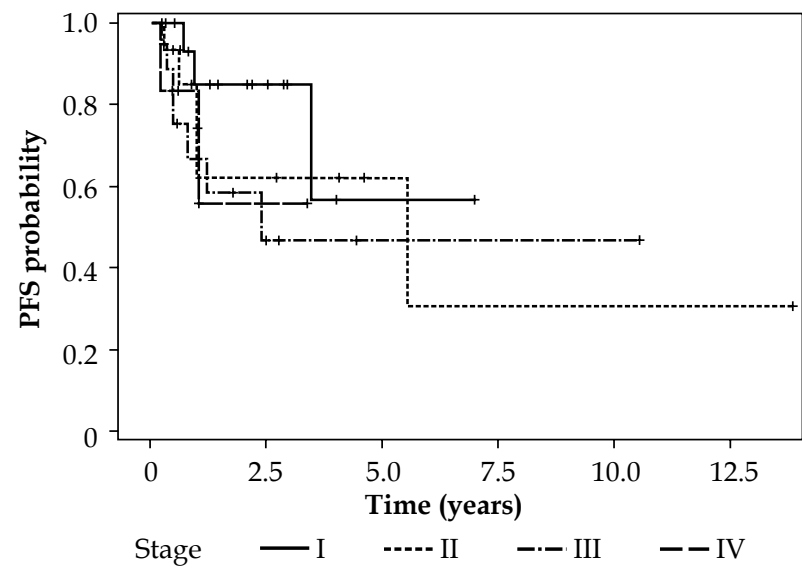

\section{Toxicity}

Acute and late toxicities were graded from 0-5, according to CTCAE v. 4.0, with late toxicities defined as those occurring after $\geq 6$ months. Toxicity grades $3-5$, illustrated in Table 3, were considered most clinically significant and consistently reported for the purposes of this retrospective analysis. There was 1 acute toxicity grade $\geq 3(2 \%)$, which was grade 3 genitourinary moist desquamation (patient $\mathrm{H}$ ). The most common late toxicity $\geq$ grade 3 was soft tissue radionecrosis of the vulva/vagina, which occurred in 5 patients $(8 \%)$. All cases resolved without surgery, although 4 patients $(80 \%)$ received hyberbaric oxygen therapy.

The remaining cases of late toxicity included -1 vaginal cancer patient with rectovaginal fistula (grade 4) requiring colostomy, and 1 cervical cancer patient with urethral stenosis (grade 3 ) requiring chronic catheterization. There were no cases of grade 5 toxicity. The limited available dosimetry, such as bladder and rectum constraints, was not sufficient for further analysis into correlates of severe toxicities (see Table 3 ). The EQD 2 doses $(\alpha / \beta$ ratio of 3 for late effects) for bladder and rectum doses were found to be acceptable. The exception was patient $C$ (bladder dose $89.7 \mathrm{~Gy}$ ), where toxicity is partially attributed to prior EBRT for colorectal cancer prior to treatment for recurrent uterine carcinosarcoma.

\section{B}

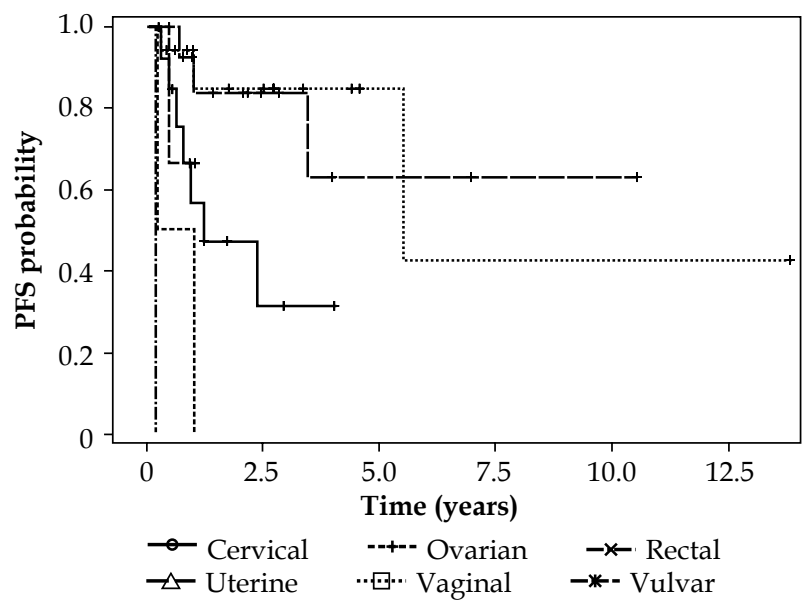

Fig. 2. Progression-free survival in patients receiving interstitial brachytherapy according to primary or recurrent tumors (A), primary site (B), and stage (I-IV) (C) 
A

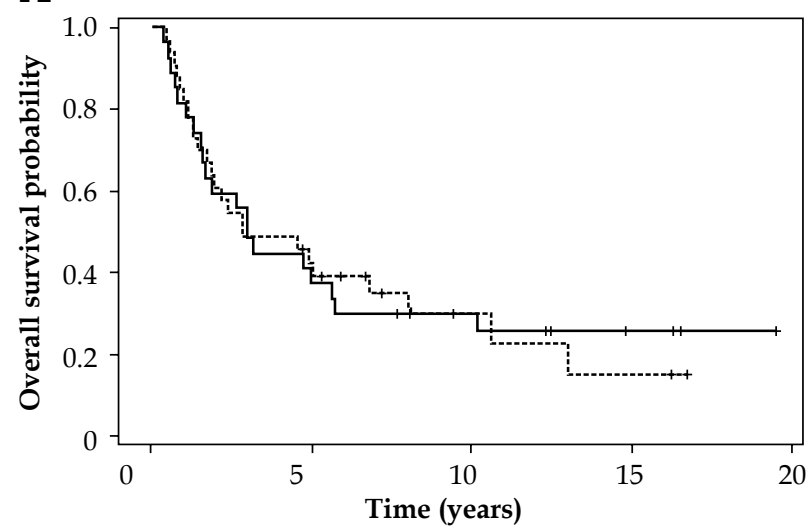

---- Primary $\rightarrow$ Recurrent

C

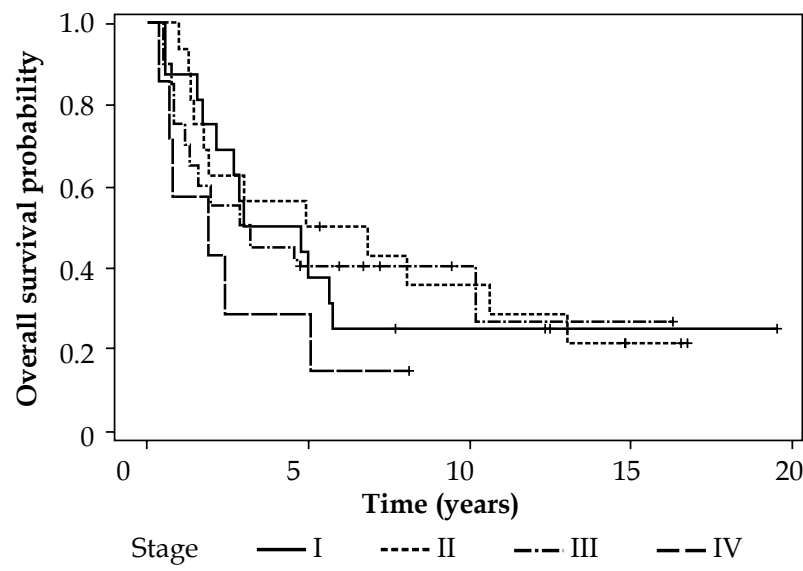

B

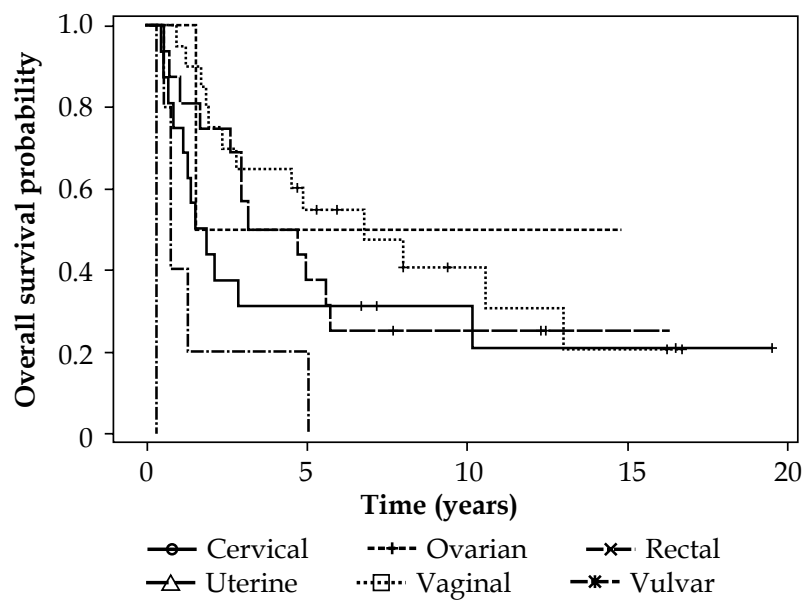

Fig. 3. Overall survival in patients receiving interstitial brachytherapy according to primary or recurrent tumors (A), primary site (B), and stage (I-IV) (C)

Table 3. Patient characteristics for cases of toxicity $\geq$ grade 3

\begin{tabular}{|c|c|c|c|c|c|c|c|}
\hline Patient & Primary & $\begin{array}{l}\text { RT, EBRT + } \\
\text { BT (Gy) }\end{array}$ & $\begin{array}{c}\text { Previous } \\
\text { RT }\end{array}$ & Grade & Toxicity & Resolution & Dosimetry, EQD2 (Gy) \\
\hline A & Vagina & $45+20$ & No & 3 & STRN, vulva & Resolved, HBO & $\begin{array}{l}\text { Bladder } D_{2 c c}=59 \\
\text { Rectum } D_{2 c c}=53\end{array}$ \\
\hline B & Vagina & $45+20$ & No & 3 & STRN, vagina & $\begin{array}{l}\text { Resolved, } \\
\text { narcotics }\end{array}$ & None available \\
\hline C & $\begin{array}{c}\text { Uterus, } \\
\text { vaginal } \\
\text { recurrence }^{a}\end{array}$ & 30 (BT only) & $\begin{array}{l}\text { Yes, } \\
64.8 \mathrm{~Gy}\end{array}$ & 3 & STRN, introitus & Resolved, HBO & $\begin{array}{c}\text { Bladder } \max =109 \\
\text { Rectum } \max =71\end{array}$ \\
\hline D & $\begin{array}{c}\text { Uterus, } \\
\text { vaginal } \\
\text { recurrence }\end{array}$ & $45+20$ & No & 3 & STRN, vagina & Resolved, HBO & Rectum $D_{2 c c}=55$ \\
\hline$E$ & $\begin{array}{l}\text { Cervix, } \\
\text { vaginal } \\
\text { recurrence }\end{array}$ & $45+25$ & No & 3 & STRN, vulva & Resolved, HBO & None available \\
\hline $\mathrm{F}$ & Vagina & $45+20$ & No & 4 & $\begin{array}{l}\text { Fistula, } \\
\text { rectovaginal }\end{array}$ & $\begin{array}{l}\text { Resolved, perma- } \\
\text { nent colostomy }\end{array}$ & None available \\
\hline$G$ & Cervix & $63+10$ & No & 3 & Urethral stricture & $\begin{array}{l}\text { Resolved, } \\
\text { chronic foley }\end{array}$ & $\begin{array}{l}\text { Bladder } D_{2 c c}=67 \\
\text { Rectum } D_{2 c c}=64\end{array}$ \\
\hline $\mathrm{H}$ & Cervix & $45+18$ & No & 3 & Skin & $\begin{array}{l}\text { Resolved, } \\
\text { hydrogel }\end{array}$ & $\begin{array}{l}\text { Bladder } \max =62 \\
\text { Rectum } \max =73\end{array}$ \\
\hline
\end{tabular}

$R T$ - radiation therapy, EBRT - external beam radiation therapy, BT - brachytherapy, EQD2 - equieffective dose in 2 Gy fractions with $\alpha / \beta=3$ for late effects, STRN - soft tissue radionecorosis, $H B O$ - hyberbaric oxygen therapy, $D_{2 c c}$ - dose to 2 cc of bladder or rectum

apatient with prior EBRT for rectal cancer (45 Gy whole pelvis with local boost to $64.8 \mathrm{~Gy}$ ), who subsequently was treated with BT only (30 Gy) for vaginal recurrence of uterine carcinosarcoma. Dosimetry data represent sum of previous and current radiation doses 


\section{Previous pelvic radiation}

Six patients received interstitial BT (median dose: $20 \mathrm{~Gy}$, range: 16-22.6 Gy) for treatment of recurrent cancers in the setting of prior pelvic EBRT. The median previous EBRT dose was 50.4 Gy (range: 45-64.8 Gy). Five patients $(83 \%)$ received interstitial BT alone without repeating EBRT, while 1 patient with stage IV rectal cancer was treated with additional 30 Gy EBRT followed by 16 Gy (4 Gy x 4) interstitial BT boost. The overall local control was $67 \%$. There were 2 local failures, which included the above rectal cancer patient who had persistent disease, as well as a patient with recurrent vulvar cancer with local relapse that expired at 9.5 months. There were 2 distant recurrences and both patients expired (18 and 36 months) with maintained local control. These patients included a recurrent uterine carcinosarcoma and a recurrent cervical adenocarcinoma. Two patients with recurrent endometrial adenocarcinoma had no evidence of disease with survival of 38 months and 195 months from BT.

\section{Discussion}

Interstitial BT is an integral component in the management of gynecologic malignancies, particularly where surgical resection would be associated with unacceptable morbidity. The American Brachytherapy Society consensus guidelines are an excellent resource for treatment of vaginal cancer with interstitial BT [7], however there is less available data to guide interstitial BT for other pelvic malignancies [14]. Retrospective series are expectedly varied with regard to patient population, tumor type, and fractionation schemes but provide important information when prospective evaluations are rare [15]. This is also a limitation of the present study, where a diverse population of primary tumors is included. In addition, comprehensive dosimetry review is not possible. The strengths of our study include: standardized BT technique with all procedures performed by one radiation oncologist $(\mathrm{SH})$, successful integration of interstitial BT as a boost after EBRT (often with chemotherapy), and a median follow-up duration of 37 months to accurately measure outcomes. Presently, we report good efficacy and favorable tolerability of interstitial BT with particular attention to 3 common clinical scenarios - locally advanced vaginal cancer, recurrent endometrial cancer, and recurrence after prior radiation. These results in the context of reported literature are further discussed below.

In our series of 20 patients with vaginal primaries receiving EBRT (85\% with concurrent chemotherapy) and interstitial BT, the 5-year OS and PFS were 55\% and $84 \%$, respectively. A comparable series of 12 patients from the University of Ottawa with primary vaginal cancer stage II-IV received EBRT with concurrent cisplatin followed by BT, of which 10 received interstitial BT [16]. The 5 year OS and PFS were $66 \%$ and $75 \%$, respectively, and 2 patients $(17 \%)$ required surgery for late toxicity, which are similar to our present series where in one patient $(5 \%)$ colostomy was required due to a rectovaginal fistula. A series from University of Vienna included 55 patients with stage II-IV vaginal cancer who received HDR-BT in com- bination with EBRT with good results. The 5-year OS for stage II and III disease was $41 \%$ and $43 \%$, and the 5 -year recurrence free survival of $50 \%$ and $23 \%$, respectively. While these are important benchmarks for comparison, the majority of patients were treated with vaginal cylinder application as opposed to interstitial BT, which the authors state likely contributed to inadequate dosing and subsequent treatment failures. Improved outcomes with interstitial BT for locally advanced vaginal cancer, in comparison to intracavitary BT, have been described [17]. In addition, concurrent chemotherapy during EBRT was not given in the Vienna study or in other vaginal cancer series treated with primary RT $[17,18]$. We attribute our favorable outcomes in vaginal cancer patients to the ability of interstitial catheters to provide adequate dose to the vagina and paravaginal tissues, and we will continue to use interstitial BT boost following chemoradiation in these patients.

Vaginal recurrences of endometrial cancer, although less common after adjuvant RT, occur in both previously non-irradiated and irradiated patients $[19,20]$. In our series that included 13 patients with endometrial cancer receiving interstitial BT without prior RT, there were no local failures seen. In an analysis of 35 patients with isolated vaginal recurrence on the PORTEC- 1 trial who thereafter were treated with curative intent, long term control was achieved in $68 \%$ [4]. Treatment of vaginal relapse most commonly included EBRT plus BT. A series from MD Anderson Cancer Center of 91 patients with isolated vaginal recurrence of endometrial carcinoma after hysterectomy included 57 patients $(63 \%)$ with recurrence after surgery alone [5]. Of the 34 who had prior RT, only 13 included EBRT with most receiving preoperative intracavitary BT. With definitive treatment for relapse including combined EBRT and BT (57\%), BT alone (12\%), or EBRT alone (31\%), the 5 -year LC and OS were $75 \%$ and $43 \%$, respectively. On multivariate analysis, combination EBRT and BT was associated with improved LC. In patients receiving BT, only $14(27 \%)$ included interstitial BT, which was given for residual disease $0.5 \mathrm{~cm}$ thick or involving the distal vagina. In 13 patients from Ohio State University receiving interstitial LDR-BT for isolated vaginal recurrence, no patients had prior RT and $85 \%$ had combination EBRT plus BT [21]. There were no cases of local failure and 3 (23\%) distant relapses were observed. These outcomes, in addition to the present series, suggest use of interstitial BT should be strongly considered for management of isolated vaginal recurrence of endometrial cancer. This is of particular importance for tumors $\geq 0.5 \mathrm{~cm}$ thick where intracavitary cylinder BT tumor dose coverage would be inadequate [7].

For recurrent pelvic tumors, interstitial BT permits effective tumor dose delivery while sparing nearby critical organs that have been previously irradiated [22]. In a series from University of Louisville that included 21 patients receiving interstitial BT for recurrent previously irradiated gynecologic tumors, the 2-year local control was $72 \%$. There were no cases of grade $4-5$ toxicities, although rates of grade 3 vaginal, urinary, and rectal toxicity were $29 \%, 9.5 \%$, and $19 \%$, respectively. Investigators from Osaka University followed 52 patients treated with reirradi- 
ation using interstitial BT for recurrent cervical cancer, with local control achieved in $77 \%$ and grade 3-4 late toxicity seen in $25 \%$. The majority (54\%) ultimately experienced death and progression of disease. This is similar to our small cohort where both local (33\%) and distant (33\%) progression are common in treating recurrent previously irradiated tumors with interstitial BT. A proportion (2 patients, 33\%) maintained local control with no evidence of disease.

\section{Conclusions}

In our series, interstitial BT most frequently following EBRT with concurrent chemotherapy was well tolerated and effective for the treatment of primary and recurrent gynecologic malignancies. There were very rare cases of late toxicity resulting in loss of pelvic organ function, which is consistent with reports in the literature. This analysis supports our continued use of interstitial BT for the treatment of gynecologic malignancies with particular focus on locally advanced vaginal, recurrent endometrial, and recurrent cancer after previous radiation. This study is an important addition to the largely retrospective published experiences on treating these patients with interstitial BT. Our current practice of HDR interstitial BT follows published recommendations for target volume coverage and reporting of both maximum organ point doses, as well dose to 2 cc volume [7,14]. Magnetic resonance imaging fusion is used in conjunction with CT-based treatment planning to aid in delineation of target volume and organs at risk, as we change to MRI simulation [23]. Our future goals include comparisons between the present cohort and subsequent patients treated with intraoperative MRI-guided BT who will have detailed dosimetry information available.

\section{Disclosure}

Authors report no conflict of interest.

\section{References}

1. Di Donato V, Bellati F, Fischetti M et al. Vaginal cancer. Crit Rev Oncol Hematol 2012; 81: 286-295.

2. Glaser SM, Beriwal S. Brachytherapy for malignancies of the vagina in the 3D era. J Contemp Brachytherapy 2015; 7: 312-318.

3. Aalders JG, Abeler V, Kolstad P. Recurrent adenocarcinoma of the endometrium: a clinical and histopathological study of 379 patients. Gynecol Oncol 1984; 17: 85-103.

4. Creutzberg CL, van Putten WL, Koper PC et al. Survival after relapse in patients with endometrial cancer: results from a randomized trial. Gynecol Oncol 2003; 89: 201-209.

5. Jhingran A, Burke TW, Eifel PJ. Definitive radiotherapy for patients with isolated vaginal recurrence of endometrial carcinoma after hysterectomy. Int J Radiat Oncol Biol Phys 2003; 56: 1366-1372.

6. Lin LL, Grigsby PW, Powell MA et al. Definitive radiotherapy in the management of isolated vaginal recurrences of endometrial cancer. Int J Radiat Oncol Biol Phys 2005; 63: 500-504.

7. BeriwalS, Demanes DJ, Erickson B et al. American Brachytherapy Society consensus guidelines for interstitial brachytherapy for vaginal cancer. Brachytherapy 2012; 11: 68-75.
8. Bahadur YA, Constantinescu C, Hassouna AH et al. Single versus multichannel applicator in high-dose-rate vaginal brachytherapy optimized by inverse treatment planning. J Contemp Brachytherapy 2015; 6: 362-370.

9. Thompson SR, Delaney GP, Gabriel GS et al. Patterns of care study of brachytherapy in New South Wales: malignancies of the uterine corpus. J Contemp Brachytherapy 2015; 7: 224-230.

10. Erickson B, Gillin MT. Interstitial implantation of gynecologic malignancies. J Surg Oncol 1997; 66: 285-295.

11. Erickson B, Albano K, Gillin M. CT-guided interstitial implantation of gynecologic malignancies. Int J Radiat Oncol Biol Phys 1996; 36: 699-709.

12. Beriwal S, Bhatnagar A, Heron DE et al. High-dose-rate interstitial brachytherapy for gynecologic malignancies. Brachytherapy 2006; 5: 218-222.

13. National Cancer Institute. Common Terminology Criteria for Adverse Events v4.0. NCI, NIH, DHHS. NIH Publication \# 09-7473. 2009.

14. Viswanathan AN, Beriwal S, De Los Santos JF et al. American Brachytherapy Society consensus guidelines for locally advanced carcinoma of the cervix. Part II: high-dose-rate brachytherapy. Brachytherapy 2012; 11: 47-52.

15. Viswanathan AN, Szymonifka J, Tempany-Afdhal CM et al. A prospective trial of real-time magnetic resonance-guided catheter placement in interstitial gynecologic brachytherapy. Brachytherapy 2013; 12: 240-247.

16. Samant R, Lau B, E C et al. Primary vaginal cancer treated with concurrent chemoradiation using Cis-platinum. Int J Radiat Oncol Biol Phys 2007; 69: 746-750.

17. Stock RG, Mychalczak B, Armstrong JG et al. The importance of brachytherapy technique in the management of primary carcinoma of the vagina. Int J Radiat Oncol Biol Phys 1992; 24: 747-753.

18. Perez CA, Grigsby PW, Garipagaoglu M et al. Factors affecting long-term outcome of irradiation in carcinoma of the vagina. Int J Radiat Oncol Biol Phys 1999; 44: 37-45.

19. Creutzberg CL, van Putten WL, Koper PC et al. Surgery and postoperative radiotherapy versus surgery alone for patients with stage-1 endometrial carcinoma: multicentre randomized trial. PORTEC Study Group. Post-Operative Radiation Therapy in Endometrial Carcinoma. Lancet 2000; 355: 1404-1411.

20. Keys HM, Roberts JA, Brunetto VL et al. A phase III trial of surgery with or without adjunctive external pelvic radiation therapy in intermediate risk endometrial adenocarcinoma: a Gynecologic Oncology Group study. Gynecol Oncol 2004; 92: 744-751.

21. Nag S, Yacoub S, Copeland LJ et al. Interstitial brachytherapy for salvage treatment of vaginal recurrences in previously unirradiated endometrial cancer patients. Int J Radiat Oncol Biol Phys 2002; 54: 1153-1159.

22. Amsbaugh MJ, Bhatt N, Hunter T et al. Computed tomography planned interstitial brachytherapy for recurrent gynecologic cancer. Brachytherapy 2015; 14: 600-605.

23. Dimopoulos JC, Petrow P, Tanderup K et al. Recommendations from Gynaecological (GYN) GEC-ESTRO Working Group (IV): Basic principles and parameters for MR imaging within the frame of image based adaptive cervix cancer brachytherapy. Radiother Oncol 2012; 103: 113-122. 